\title{
An Abstract Model for Service Provision, Search and Composition
}

\author{
Hui Ma \\ School of Engineering and \\ Computer Science \\ Victoria University of Wellington \\ Wellington, New Zealand \\ Email: hui.ma@ecs.vuw.ac.nz
}

\author{
Klaus-Dieter Schewe \\ Information Science Research Centre \\ Palmerston North, New Zealand \\ Email: kdschewe@acm.org
}

\author{
Qing Wang \\ University of Otago \\ Dunedin, New Zealand \\ Email: qing.wang@otago.ac.nz
}

\begin{abstract}
Service-oriented computing, cloud computing, and web services composition mark cornerstones of a paradigm shift in software systems engineering. The general new idea is to use as much as possible services that are made available by others, mostly disseminated via the web. In this paper, we present an abstract model for clouds as federations of services together with a specification of semantics and quality characteristics. For the services as such we adopt the abstract model of abstract state services, which is based on views on some hidden database layer that are equipped with service operations. For the semantics we adopt types for in- and output, pre- and post-conditions, and a description of functionality within an operations ontology. In addition, quality characteristics capture performance, costs, availability, etc. On the basis of this model of clouds, users may conduct a (web) search for usable services, extract service components, and recompose these components. The quality characteristics can be used to optimise the selection of usable services.
\end{abstract}

\section{INTRODUCTION}

A lot of research is currently investigated into serviceoriented architectures (SOA) (see e.g. [7], [10]), serviceoriented computing (SOC), web services (see e.g. [2], [4], [8]), and cloud computing, which are all centred around related problems. A quick search on the DBLP bibliography server reveals that in less than a decade nearly 500 articles were published with keywords "service-oriented" and "architecture" in the title, not counting the far larger number of papers dealing with the subject, but not having it already in the title.

Despite this big interest in the area, and the many ideas and systems that have been created many fundamental questions have still not been answered. For instance, a web service can be almost anything, a simple function, a data warehouse, or a fully functional Web Information System, as long as it is made available via the world-wide web. The unifying characteristic is that content, functionality and sometimes even presentation are made available for use by human users or other services. This view of a pool of resources is also used in the meme media architecture [16], which is based on research that started in the second half of the 1980s, and thus, being older than the world-wide-web is not restricted to the web. The general idea is that media resources are extracted from any accessible source, wrapped and thereby brought into the generic form of a meme media object, and stored in a meme pool, from which they can be retrieved, re-edited, recombined, and redistributed.

In an effort to consolidate and integrate current research activities, the Service-Oriented Computing Research Roadmap [13] has been proposed. Service foundations, service composition, service management and monitoring, and service-oriented engineering have been identified as core SOC research themes. Our research aims at laying the foundations of a theory of service-oriented systems. In particular, we try to answer the following fundamental questions:

- How must a general model for services look like capturing the basic idea and all facets of possible instantiations, and how can we specify such services?

- How can we search for services that are available on the web?

- How do we extract from such services the components that are useful for the intended application, and how do we recombine them?

- How can we optimise service selection using functional and non-functional (aka "quality of service") criteria?

In this paper we deal with the first two of these questions. We first present the model of Abstract State Services (ASSs) as a general, formal model for services. It is based on Abstract State Machines (ASMs) [6], which have already been shown their usefulness in many areas, e.g. formalising the notion of sequential and parallel algorithm [5], [9], and modelling web services [1]. ASSs abstract from and generalise our research on Web Information Systems [14], and integrate the customised ASM thesis for database transformations [15]. ASSs were introduced in [11], and are described in detail in [12] including their composition.

ASSs give an answer to the first problem above and partly to the third one. Searching for services, however, requires additions to the model. For this we pick up the idea that a "cloud" (or similarly, a "meme pool") provides a collection of services. Thus, we propose a formalisation of clouds by federations of services, which in addition provide a description of the offered services. Such a description can be given in form of an ontology. It must contain a technical description of services by means of types, and pre- and post-conditions, but this is 
not yet sufficient, as the application area and functionality of the service must also be described by keywords. In a sense, this is the idea of the "semantic web", which enables at least the semi-automatic selection of services. In order to become fully automatic, the terminologies used by the service provider and the service seeker must match, for which service brokers may be a suitable idea.

In the remainder of the paper we will elaborate on ASSs, their composition, the model of clouds as service federations, and the use of the model for service discovery. We start in Section II by discussing a scenario for building a serviceoriented application to highlight, which problems have to be solved. In Section III we briefly present our model of ASSs. For details, however, we have to refer to [12]. Section IV is then devoted to clouds as service federations, i.e. the main emphasis will be on description logics for the specification of such clouds. In particular, we outline how the model can be used to search for services. We conclude with a brief summary and outlook.

\section{A Motivating Scenario}

The general scenario for (web-based) service-oriented computing is that in order to execute a task data and functionality of various services will be required. For instance, when planning a conference trip, the participant will have to choose a service for flight booking, use the registration service, book accommodation and other transport, maybe use services to arrange a touristic side program, get maps, etc. Thus, a conference planning application system has to be decomposed into components, services for some or all of these components have to be searched for, among the search results a selection has to be made, and the selected components have to be recomposed. In this case most of the needed services would be booking services, which constitute well established web information systems.

Another example would be investment planning, for which the needed services would be financial services, product comparisons, and other economic services, which are also available to a large extent.

Let us further look at the task of creating a national/regional water quality report for streams, rivers and lakes. For this we first require several geographical services about location and flow of streams, rivers and lakes, and their connections, and about locations of major water consumers and wastewater producers such as farms, cities/towns, roads, and other settlements. In addition, we require data from services dealing with water and waste water contracts, i.e. under which conditions such as water level, nitrate and oxygen concentration, concentration of harmful substances, etc. water can be taken out or waste-water can be let run in, respectively. We also require additional information about usage of farmland and models and the possible impact on the adjacent water. We require access to weather and water databases, from which we can get data about rainfall and water quality measurements. Furthermore, we require prediction models for water levels and quality attributes based on out-take, inlets, rain, etc., and assessment models classifying the predictions.

The required services are offered by farms through whole farm plans, land-care and meteorological authorities, and scientific bodies. Whether the access is free or subject to authorisation or payment is of minor interest for us.

\section{Abstract State Services}

Based on the discussion in Section II we assume that services are data-intensive, so we first look at databases. This will enable us to combine data services with functional services. The assumption of an underlying database is no real restriction, as it is hidden anyway, and data services will be formalised by views, which in the extreme case could be empty to capture pure functional services. Traditional database architecture distinguishes at least three layers: a conceptual layer describing the database schema in an abstract way, a physical layer implementing the schema, and an external layer made out of views. The external layer exports the data that can then be used by users or programs. For our purposes here we can neglect the physical layer, but in order to capture dataintensive services, we complete this architecture by adding operations on both the conceptual and the external layer, the former one being handled as database transactions, whereas the latter ones provide the means with which users can interact with a database.

\section{A. A Two-Layer Model}

Starting with the database layer and following the general approach of Abstract State Machines [9] we may consider each database computation as a sequence of abstract states, each of which represents the database (instance) at a certain point in time plus maybe additional data that is necessary for the computation, e.g. transaction tables, log files, etc. In order to capture the semantics of transactions we distinguish between a wide-step transition relation and small step transition relations. A transition in the former one marks the execution of a transaction, so the wide-step transition relation defines infinite sequences of transactions. Without loss of generality we can assume a serial execution, while of course interleaving is used for the implementation. Then each transaction itself corresponds to a finite sequence of states resulting from a small step transition relation, which should then be subject to the postulates for database transformations [15].

DEFINITION III.1 A database system DBS consists of a set $\mathcal{S}$ of states, together with a subset $\mathcal{I} \subseteq \mathcal{S}$ of initial states, a widestep transition relation $\tau \subseteq \mathcal{S} \times \mathcal{S}$, and a set $\mathcal{T}$ of transactions, each of which is associated with a small-step transition relation $\tau_{t} \subseteq \mathcal{S} \times \mathcal{S}(t \in \mathcal{T})$ satisfying the postulates of a database transformation over $\mathcal{S}$.

A run of a database system DBS is an infinite sequence $S_{0}, S_{1}, \ldots$ of states $S_{i} \in \mathcal{S}$ starting with an initial state $S_{0} \in \mathcal{I}$ such that for all $i \in \mathbb{N}\left(S_{i}, S_{i+1}\right) \in \tau$ holds, and there is a transaction $t_{i} \in \mathcal{T}$ with a finite run $S_{i}=S_{i}^{0}, \ldots, S_{i}^{k}=S_{i+1}$ such that $\left(S_{i}^{j}, S_{i}^{j+1}\right) \in \tau_{t_{i}}$ holds for all $j=0, \ldots, k-1$. 
Views in general are expressed by queries, i.e. read-only database transformations. Therefore, we can assume that a view on a database state $S_{i} \in \mathcal{S}$ is given by a finite run $S_{i}=S_{0}^{v}, \ldots, S_{\ell}^{v}$ of some database transformation $v$ with $S_{i} \subseteq S_{\ell}^{v}$ - traditionally, we would consider $S_{\ell}^{v}-S_{i}$ as the view. We can use this to extend a database system by views.

In doing so we let each state $S \in \mathcal{S}$ to be composed as a union $S_{d} \cup V_{1} \cup \cdots \cup V_{k}$ such that each $S_{d} \cup V_{j}$ is a view on $S_{d}$. As a consequence, each wide-step state transition becomes a parallel composition of a transaction and an operation that "switches views on and off". This leads to the definition of an Abstract State Service (ASS).

Definition III.2 An Abstract State Service (ASS) consists of a database system DBS, in which each state $S \in \mathcal{S}$ is a finite composition $S_{d} \cup V_{1} \cup \cdots \cup V_{k}$, and a finite set $\mathcal{V}$ of (extended) views. Each view $v \in \mathcal{V}$ is associated with a database transformation $q_{v}$ such that for each state $S \in \mathcal{S}$ there are views $v_{1}, \ldots, v_{k} \in \mathcal{V}$ with finite runs $S_{d}=S_{0}^{j}, \ldots, S_{n_{j}}^{j}=S_{d} \cup V_{j}$ of $v_{j}(j=1, \ldots, k)$. Each view $v \in \mathcal{V}$ is further associated with a finite set $\mathcal{O}_{v}$ of (service) operations $o_{1}, \ldots, o_{n}$ such that for each $i \in\{1, \ldots, n\}$ and each $S \in \mathcal{S}$ there is a unique state $S^{\prime} \in \mathcal{S}$ with $\left(S, S^{\prime}\right) \in \tau$. Furthermore, if $S=S_{d} \cup V_{1} \cup \cdots \cup V_{k}$ with $V_{i}$ defined by $v_{i}$ and $o$ is an operation associated with $v_{k}$, then $S^{\prime}=S_{d}^{\prime} \cup V_{1}^{\prime} \cup \cdots \cup V_{m}^{\prime}$ with $m \geq k-1$, and $V_{i}^{\prime}$ for $1 \leq i \leq k-1$ is still defined by $v_{i}$.

In a nutshell, in an ASS we have view-extended database states, and each service operation associated with a view induces a transaction on the database, and may change or delete the view it is associated with, and even activate other views. These service operations are actually what is exported from the database system to be used by other systems or directly by users.

Note that for each view $v$ the defining query, i.e. the database transformation $q_{v}$, can be considered itself a service operation. This simply reflects the fact that data that is made available on the web can be extracted and stored or processed elsewhere. In particular, we have the extreme cases of a pure data service, in which no service operations would be associated with a view $v$,i.e. $\mathcal{O}_{v}=\emptyset$, and a pure functional service, in which the view $v$ is empty.

A formalisation of database transformations by means of postulates is beyond the scope of this paper. In a nutshell, the postulates require a one-step transition relation between states (sequential time postulate), states as (meta-finite) first-order structures (abstract state postulate), necessary background for database computations such as complex value constructors (background postulate), limitations to the number of accessed terms in each step (bounded exploration postulate), and the preservation of equivalent substructures in one successor state (genericity postulate) [15].

\section{B. Examples}

We sketch the specification of ASSs using application scenarios from Section II.
EXAMPLE III.1 Let us look at the case of the water quality report. We already elaborated that services from farms would be needed.

For the database layer of such services we can assume that the database contains relations for geographical data such as paddocks, fences, rivers, ponds, etc., for which geometric data types such as lines, polygons, quadratic Bézier curves, etc. would be used. The database should further contain relations for water consent determining the maximum volume of outtake for specified extraction locations, and the minimum water level at these points. Furthermore, the waste-water agreement would specify minimum or maximum levels, respectively, of oxygen, nitrate, etc.

While these data are rather static the database should further contain data that change over time such as the use of paddocks (for sheep, cattle or crop) and the use of water for irrigation.

On these grounds a view could be built for a water consumption statistics, i.e. in which month at which location point was how much water extracted, and how was it used, e.g. for irrigation at several paddocks or for animal watering stations. The statistics could further indicate the minimum and maximum water level of the river, the water was taken from.

Service operations could be defined for slicing out only part of the data, widening or narrowing the observation window (from months to days or quarters), to build aggregations, to relate the data to the average permitted out-take according to the water consent, etc. For the purpose of the serviceseeking task, the water quality report, these functional service operations are of minor importance. What is mainly needed from the farms is the data service, i.e. the view defining queries.

Now look at the subtask of the service seeking task to estimate water levels and predict the water quality for a river $r$ at a specific measurement location $A$. For this, we would first require to know all upstream tributaries of the river, which can be extracted from a different global geographic service, and compute all farms that have extraction locations associated with the river upstream $A$ or any of the tributaries.

Then the data extracted from these farms by means of the defining queries for water consumption statistics can be fed into an operation to compute the water level at $A$ within some boundaries - of course, rainfall extracted from elsewhere has also to be taken into account. This allows to create a new view with monthly listed, predicted and measured water levels at $A$. A service operation associated with this view could be a prediction of water quality properties for a certain time period, which would also use data extracted from farm data (using a different view based on waste-water inlet). This service operation can be further composed with an analytic operation, which detects the potential source for critical quality values. This service operation would have to make use of the usage data obtained from farms.

Example III.1 is heavy on the use of data as a service, but does not exploit many functional service operations. The following example illustrates a case, where functionality is 
used more intensively.

EXAMPLE III.2 As emphasized in the previous section, the task to create a conference trip preparation system requires the use of several booking systems. For instance, a flight booking service requires data of origin and destination, which are used to create a view with a list of possible connections including departure and arrival time, number of stops, total duration, costs, etc. A typical service operation associated with this view is to select one of the flights and to book them.

The booking service for accommodation is similar, yet the composition requires compatibility of dates.

\section{Clouds as Service Federations}

The common understanding of the notion of "cloud" is that it is some kind of service pool, from which services can be extracted and used. Thus, one of the key problems is to discover the services that are needed for a particular application. This should be possible by means of a search engine. Therefore, it is crucial that the service operations including the view defining queries that are made available through some cloud are provided with an adequate description, which will allow a search engine to discover (with some certainty) the required services.

Such a description should at least comprise three parts:

- a functional description of input- and output types as well as pre- and post-conditions telling in technical terms, what the service operation will do,

- a categorical description by inter-related keywords telling what the service operation does by using common terminology of the application area, and

- a quality of service (QoS) description of non-functional properties such as availability, response time, cost, etc.

The QoS description is not needed for service discovery and merely useful to select among alternatives, but neither functional nor categorical description can be dispensed with.

A functional description alone would be insufficient. For instance, a flight booking service operation as in Example III.2 requires an itinerary to be selected, so the input type could be specified as $\{$ (flight_no : STRING, day : DATE, departure : TIME, class : CHAR, price : $D E C I M A L)\}$, i.e. the input is a finite set of tuples, each of which defines a flight number, departure day and time, the booking class and the price. The output type could be similar with a status (confirmed, waitlisted, unavailable) added for each flight segment, i.e. we have the type $\{$ (flight_no : STRING, day : DATE, departure : TIME, class : CHAR, price : DECIMAL, status : STRING)\}. A precondition could simply be that the selected itinerary is meaningful, i.e. flight numbers exist for the corresponding date and time, and are compatible. However, no meaningful post-condition can be specified, as the output depends on the status of the (hidden) flight database. Moreover, a booking service for railway tickets would require the same types, so the functional description does not indicate exactly what kind of service is offered.
As for the categorical description, the terminology has to be specified. This defines an ontology in the widest sense, i.e. we have to provide definitions of "concepts" and relationships between them, such that each offered service becomes an instantiation of one or several concepts in the terminology. In this way we adopt the fundamental idea of the "semantic web".

In the following we will outline how ontologies can be exploited for service description. We will pay particular attention to description logics without prescribing a particular language. That is, OWL [17] or more expressive description logics [3] could be exploited.

\section{A. Ontologies}

As outlined, the functional, categorical and QoS description of services in a cloud requires the definition of an ontology. That is, we need a terminological knowledge layer (aka TBox in description logics) describing concepts and roles (or relationships) among them. This usually includes a subsumption hierarchy among concepts (and maybe also roles), and cardinality constraints. In addition, there is an assertional knowledge layer (aka ABox in description logics) describing individuals. Thus, services in a cloud constitute the ABox of an ontology, while the cloud itself is defined by the TBox.

In principle, instead of TBox and ABox we could use the more classical notions of schema and instance, and exploit any kind of data model. A query language associated with the used data model, could then be used to find the required services. In fact, description logics only provide rather limited logics with respect to expressiveness. There are two major reasons for giving preference to description logics:

1) Description logics use two important relationships, which due to the restrictions become decidable ${ }^{1}$ : subsumption and instantiation. Subsumption is a binary relationship between concepts (denoted as $C_{1} \sqsubseteq C_{2}$ ) guaranteeing that all instances of the subsumed concept $C_{1}$ are also instances of the subsuming concept $C_{2}$. Instantiation defines a binary relationship between instances in the ABox and concepts in the TBox asserting that an element $A$ of the ABox is an instance of a concept $C$ in the TBox.

Subsumption and instantiation together allow us to discover services that are more expressive than needed, but can be projected to a service just as required.

2) Concept and role names in the TBox can be subject to similarity search by a search engine. That is, the search engine could produce services that are similar (with a certainty factor) to the ones required with respect to the categorical description, and match the functional description.

Let us now look more closely into one particular description logic in the DL-Lite family (see [3]). For this assume that $C_{0}$

\footnotetext{
${ }^{1}$ This is not completely true for all description logics. There are more expressive description logics (see [3]) that come with the price of losing decidability of subsumption in the general case.
} 
and $R_{0}$ represent not further specified specified sets of basic concepts and roles, respectively. Then concepts $C$ and roles $R$ are defined by the following grammar:

$$
\begin{aligned}
& R=R_{0} \mid R_{0}^{-} \\
& A=C_{0}|\top| \geq m . R(\text { with } m>0) \\
& C=A|\neg C| C_{1} \sqcap C_{2}\left|C_{1} \sqcup C_{2}\right| \exists R . C \mid \forall R . C
\end{aligned}
$$

Definition IV.1 A terminology (or TBox) is a finite set $\mathcal{T}$ of assertions of the form $C_{1} \sqsubseteq C_{2}$ with concepts $C_{1}$ and $C_{2}$ as defined by the grammar above.

Each assertion $C_{1} \sqsubseteq C_{2}$ in a terminology $\mathcal{T}$ is called a subsumption axiom. Note that Definition IV.1 only permits subsumption between concepts, not between roles, though it is possible to define more complex terminologies that also permit role subsumption.

As usual, we use the shortcut $C_{1} \equiv C_{2}$ instead of $C_{1} \sqsubseteq$ $C_{2} \sqsubseteq C_{1}$. For concepts, $\perp$ is a shortcut for $\neg \top$, and $\leq m . R$ is a shortcut for $\neg \geq m+1 . R$.

DEFINITION IV.2 A structure $\mathcal{S}$ for a terminology $\mathcal{T}$ consists of a non-empty set $\mathcal{O}$ together with subsets $\mathcal{S}\left(C_{0}\right) \subseteq \mathcal{O}$ and $\mathcal{S}\left(R_{0}\right) \subseteq \mathcal{O} \times \mathcal{O}$ for all basic concepts $R_{0}$ and basic roles $R_{0}$, respectively. $\mathcal{O}$ is called the base set of the structure.

We first extend the interpretation of basic concepts and roles and to all concepts and roles as defined by the grammar above, i.e. for each concept $C$ we define a subset $\mathcal{S}(C) \subseteq \mathcal{O}$, and for each role $R$ we define a subset $\mathcal{S}(R) \subseteq \mathcal{O} \times \mathcal{O}$ as follows:

$$
\begin{gathered}
\mathcal{S}\left(R_{0}^{-}\right)=\left\{(y, x) \mid(x, y) \in \mathcal{S}\left(R_{0}\right)\right\} \\
\mathcal{S}(\top)=\mathcal{O} \\
\mathcal{S}(\geq m . R)=\{x \in \mathcal{O} \mid \#\{y \mid(x, y) \in \mathcal{S}(R)\} \geq m\} \\
\mathcal{S}(\neg C)=\mathcal{O}-\mathcal{S}(C) \\
\mathcal{S}\left(C_{1} \sqcap C_{2}\right)=\mathcal{S}\left(C_{1}\right) \cap \mathcal{S}\left(C_{2}\right) \\
\mathcal{S}\left(C_{1} \sqcup C_{2}\right)=\mathcal{S}\left(C_{1}\right) \cup \mathcal{S}\left(C_{2}\right) \\
\mathcal{S}(\exists R . C)=\{x \in \mathcal{O} \mid(x, y) \in \mathcal{S}(R) \text { for some } y \in \mathcal{S}(C)\} \\
\mathcal{S}(\forall R . C)=\{x \in \mathcal{O} \mid(x, y) \in \mathcal{S}(R) \Rightarrow y \in \mathcal{S}(C) \text { for all } y\}
\end{gathered}
$$

DEFINITION IV.3 A model for a terminology $\mathcal{T}$ is a structure $\mathcal{S}$, such that $\mathcal{S}\left(C_{1}\right) \subseteq \mathcal{S}\left(C_{2}\right)$ holds for all assertions $C_{1} \sqsubseteq C_{2}$ in $\mathcal{T}$. A finite model, i.e. a model with a finite base set, is also called instance or ABox associated with $\mathcal{T}$.

EXAMPLE IV.1 The general part of a service ontology could be defined by a terminology as follows:

$$
\begin{gathered}
\text { Service } \sqsubseteq \exists \text { name.Identifier } \sqcap \leq 1 \text {.name } \sqcap \exists \text { address.URL } \sqcap \\
\exists \text { offered_by.Provider } \sqcap \leq 1 \text {.address } \sqcap \leq 1 \text {.offered_by } \\
\sqcap \exists \text { defining.Query } \sqcap \leq 1 \text {.defining } \sqcap \exists \text { offers.Operation } \\
\text { Operation } \sqsubseteq \exists \text { associated_with.Query } \sqcap \leq 1 \text {.associated_with } \\
\text { Data_Service } \equiv \text { Query } \sqcap \geq 1 \text {.defining }{ }^{-} \\
\text {Functional_Service } \equiv \text { Operation } \sqcap \geq 1 \text {.offers }{ }^{-} \\
\text {Service_Operation } \equiv \text { Data_Service } \sqcup \text { Functional_Service } \\
\text { Service_Operation } \sqsubseteq \exists \text { input.Type } \sqcap \leq 1 \text {.input } \\
\exists \text { output.Type } \sqcap \leq 1 \text {.output } \\
\text { Type } \sqsubseteq \exists \text { name.Identifier } \sqcap \leq 1 \text {.name } \sqcap \exists \text { format.Format }
\end{gathered}
$$

Here we used capital first letters to indicate concept names, and lower case letters for role names.

\section{B. Clouds and Trusted Service Brokers}

Following our discussion above we can now formally define a cloud as a federation of services together with a descriptive ontology.

Definition IV.4 A cloud is a finite collection $\left\{\mathcal{A}_{i} \mid i \in I\right\}$ of ASSs together with a terminology $\mathcal{T}$, such that the defining queries of views and the associated service operations of these ASSs define an instance of $\mathcal{T}$.

In principle, this definition permits any kind of terminology, as long as it deals with service operations. However, as outlined above we expect the terminology $\mathcal{T}$ of a cloud to provide the functional, categorical and QoS description of its offered services.

a) Functional Description.: The functional description of a service operation consists of input- and output-types as already indicated in Example IV.1, and pre- and postconditions. For the types we need a type system with base types and constructors. For instance, the following grammar

$$
\begin{aligned}
t=b|\mathbb{1}|\left(a_{1}: t_{1}, \ldots, a_{n}: t_{n}\right)|\{t\}|[t] \mid \\
\left(a_{1}: t_{1}\right) \oplus \cdots \oplus\left(a_{n}: t_{n}\right)
\end{aligned}
$$

describes (the abstract syntax of) a type system with a trivial type 1 , a non-further specified collection of base types $b$, and four type constructors $(\cdot)$ for record types, $\{\cdot\}$ for finite set types, $[t]$ for list types, and $\oplus$ for union types. Record and union types use field labels $a_{i}$.

The semantics of such types is basically described by their domain, i.e. sets of values $\operatorname{dom}(t)$. Usually, for a base type $b$ such as Cardinal, Decimal, Float, etc. the domain is some commonly known at most countable set with a common presentation. The domain of the trivial type contains a single special value, say $\operatorname{dom}(\mathbb{1})=\{\perp\}$. For constructed types we 
obtain the domain in the usual way:

$$
\begin{aligned}
& \operatorname{dom}\left(\left(a_{1}: t_{1}, \ldots, a_{n}: t_{n}\right)\right)= \\
& \left\{\left(a_{1}: v_{1}, \ldots, a_{n}: v_{n}\right) \mid a_{i} \in \operatorname{dom}\left(t_{i}\right) \text { for } i=1, \ldots, n\right\} \\
& \operatorname{dom}(\{t\})=\{A \mid A \subseteq \operatorname{dom}(t) \text { finite }\} \\
& \operatorname{dom}([t])=\left\{\left[v_{1}, \ldots, v_{k}\right] \mid v_{i} \in \operatorname{dom}(t) \text { for } i=1, \ldots, k\right\} \\
& \operatorname{dom}\left(\left(a_{1}: t_{1}\right) \oplus \cdots \oplus\left(a_{n}: t_{n}\right)\right)= \\
& \qquad \bigcup_{i=1}^{n}\left\{\left(a_{i}: v_{i}\right) \mid v_{i} \in \operatorname{dom}\left(t_{i}\right)\right\}
\end{aligned}
$$

In particular, a union type $\left(a_{1}: \mathbb{1}\right) \oplus \cdots \oplus\left(a_{n}: \mathbb{1}\right)$ has the domain $\left\{\left(a_{1}: \perp\right), \ldots,\left(a_{n}: \perp\right)\right\}$, which can be identified with the set $\left\{a_{1}, \ldots, a_{n}\right\}$, i.e. such types are in fact enumeration types.

It is no problem to add the specification of types to the general service terminology as outlined in Example IV.1 thereby defining part of the functional description.

EXAMPLE IV.2 We can extend the terminology in Example IV.1 by the following axioms for types:

$$
\begin{gathered}
\text { Type } \equiv \text { Base_type } \sqcup \text { Trivial_type } \sqcup \text { Composed_type } \\
\text { Composed_type } \equiv \text { Record } \sqcup \text { Set } \sqcup \text { List } \sqcup \text { Union } \\
\text { Record } \sqsubseteq \forall \text { component.Field } \\
\text { Field } \sqsubseteq \exists \text { field_name.Identifier } \sqcap \leq 1 \text {.field_name } \\
\sqcap \exists \text { type.Type } \sqcap \leq 1 \text {.type } \\
\text { Union } \sqsubseteq \forall \text { component.Field } \\
\text { Record } \sqcap \text { Union } \sqsubseteq \perp \\
\text { Set } \sqsubseteq \exists \text { component.Type } \sqcap \leq 1 \text {.component } \\
\text { List } \sqsubseteq \exists \text { component.Type } \sqcap \leq 1 \text {.component } \\
\text { Set } \sqcap \text { List } \sqsubseteq \perp
\end{gathered}
$$

Of course, the specification of composed types impacts directly on the format, which is defined by field names and the format for the component type(s). Nevertheless, this constraint can be handled by the specification of ABox assertions.

In addition to the types, the functional description of a service operation includes pre- and post-conditions, which are defined by (first-order) predicate formulae. These formulae may contain further functions and predicates, which are subject to further (categorical) description.

EXAMPLE IV.3 The terminology in Examples IV.1 and IV.2 can be further extended by the following axioms:

$$
\begin{gathered}
\text { Service_Operation } \sqsubseteq \forall \text { pre.Condition } \sqcap \leq 1 \text {.pre } \\
\qquad \exists \text { post.Condition } \sqcap \leq 1 \text {.post } \\
\text { Condition } \sqsubseteq \text { Formula } \sqcap \forall \text { uses.(Predicate } \sqcap \text { Function) } \\
\text { Predicate } \sqsubseteq \exists \text { in.Type } \sqcap \leq 1 \text {.in } \sqcap \neg \geq 1 \text {.out } \\
\text { Function } \sqsubseteq \exists \text { in.Type } \sqcap \leq 1 \text {.in } \sqcap \exists \text { out.Type } \sqcap \leq 1 \text {.out }
\end{gathered}
$$
$\diamond$

This would complete the functional part of the terminology. b) Quality of Service.: Quality of service properties are availability, cost, response time, etc. These can be added directly by means of roles to the concept Service_Operation.

EXAMPLE IV.4 By means of the axiom

Service_Operation $\sqsubseteq \exists$ cost.Amount $\sqcap \exists$ availability.Times $\sqcap$ response_time.Duration

the terminology in Example IV.1 will be extended by a QoS description.

c) Categorical Description.: As shown at the beginning of this section, the functional description is insufficient for enabling service discovery, and the QoS description is only needed as a means to support the selection among several alternatives. The core of the service description by means of the terminology of a cloud is the categorical description, which refers to the standard terminology of the application area, and relates the used notions to each other.

There are no general requirements for the categorical description, as it depends completely on the application domain. However, it will always lead to subconcepts of the concept Service_Operation plus additional concepts and roles. It will also add more details to the predicates and functions used in the pre- and post-conditions.

EXAMPLE IV.5 Let us look again at booking services as required by the conference trip application in Example III.2 with particular emphasis on flight booking. The categorical description may consist of the following axioms:

$$
\begin{gathered}
\text { Booking } \sqsubseteq \text { Service_Operation } \sqcap \exists \text { initiator.Customer } \sqcap \\
\exists \text { initiated_by.Request } \sqcap \exists \text { receives.Acknowledgement } \\
\sqcap \exists \text { requires.Customer_data } \sqcap \exists \text { requires.Payment } \sqcap \\
\exists \text { receives.(Confirmation } \sqcup \text { Declination } \sqcup \text { Amendment) } \\
\text { Request } \sqsubseteq \exists \text { object.Booking_object } \sqcap \exists \text { date.DATE } \\
\text { Flight_booking } \sqsubseteq \text { Booking } \sqcap \forall \text { initiated_by.Flight_request } \\
\text { Flight_request } \sqsubseteq \text { Request } \sqcap \forall \text { object.Flight } \\
\text { Flight } \sqsubseteq \text { Booking_object } \sqcap \exists \text { number.Flight_number } \sqcap \\
\exists \text { carrier.Airline } \sqcap \exists \text { departure.Date } \sqcap \text { duration.Duration } \\
\square \exists \text { origin.Airport } \sqcap \exists \text { destination.Airport }
\end{gathered}
$$

This specification is of course incomplete, but it shows how to proceed. That is, a booking is defined by a service operation that is initiated by a request from a customer, it further requires customer data and payment, and leads to an acknowledgement plus a confirmation, declination or (suggested) amendment. The request for a booking contains at least a booking object it could contain more than one - and a date. A flight booking is a booking that is initiated by a flight request, which is a request, in which all booking object are flights. Flights themselves must have at least a flight number, an airline, a departure date, a duration, and origin and destination airports. $\diamond$ 
The terminology of a cloud enables the discovery of services, as it can be queried. We will look at this in more detail in the following section. However, the success of a search depends on the compatibility of the ontologies used by the service seeker and the service provider. If there is no common understanding of the terms used in the terminology, in particular in the categorical description, the service seeker may apply a service, which does not deliver the required functionality.

This problem can be addressed by integrating and aligning terminologies of various clouds, which simply leads to a larger cloud. By adopting a common terminology, service providers demonstrate trust in the provider of the integrated cloud. We therefor call such integrated clouds trusted service brokers (TSBs). Furthermore, service seekers may subscribe to a TSB and thus have access to its terminology, so that queries can be formulated in advance in an adequate way.

\section{Examples}

Let us now look at the ABox of a terminology in a cloud or TSB, respectively. We use again our booking example.

EXAMPLE IV.6 Let us assume that the terminology of a cloud is defined as sketched in Examples IV.1 - IV.5. That is, the cloud provides among others flight booking services. The detailed description of such services is provided by the ABox, which can be defined by the several assertions. The general description gives rise to the following assertions.

$$
\begin{gathered}
\operatorname{Service}\left(s_{23}\right) \\
\operatorname{name}\left(s_{23}, \text { "flight booking service } 23\right. \text { ") } \\
\text { Identifier("flight booking service } 23 ") \\
\operatorname{URL}(\text { www.houseoftravel.com/fbs23) } \\
\text { address }\left(s_{23},\right. \text { www.houseoftravel.com/fbs23) } \\
\text { Provider(House of Travel) } \\
\text { offered_by }\left(s_{23}, \text { House of Travel }\right) \\
\text { Query }\left(q_{23}\right) \\
\text { defining }\left(s_{23}, q_{23}\right) \\
\text { Functional_Service }\left(\text { op }_{237}\right) \\
\text { offers }\left(s_{23}, \text { op }_{237}\right) \\
\text { associated_with }\left(\text { op }_{237}, q_{23}\right) \\
\text { input }\left(\text { op }_{237}, \text { FlightRecordSet }\right) \\
\text { output }\left(\text { op }_{237}, \text { FlightRecordStatusSet }\right)
\end{gathered}
$$

The input type is specified by the following assertions about types (omitting type names and formats):

$$
\begin{gathered}
\text { Set }(\text { FlightRecordSet }) \\
\text { component }(\text { FlightRecordSet }, \text { FlightRecord }) \\
\operatorname{Record}(\text { FlightRecord }) \\
\text { component }(\text { FlightRecord }, \text { FlightNumber })
\end{gathered}
$$

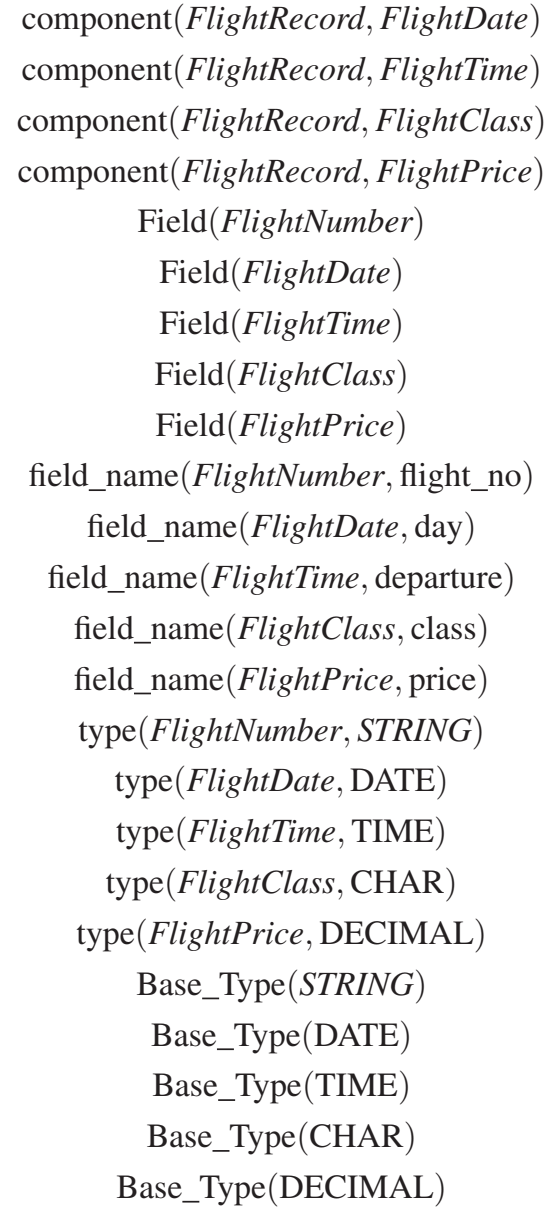

The assertions for the output type are analogous, as only one additional field is added. We also omit pre- and postconditions.

Finally, the categorical description of the flight booking service may give rise to the following assertions:






\section{reference("flight booking confirmed", $f_{23}$ )}

Confirmation("flight booking confirmed")

This ABox definition is of course also rather sketchy and incomplete, but it also indicates, how the ABox is specified, which determines of functional, categorical and (optional) QoS descriptions.

\section{Searching in the Clouds}

We mentioned above that the terminology of a cloud (or TSB) can be searched. For this we assume that the terminology $\mathcal{T}$ is complete with respect to subsumption, i.e. whenever $C_{1} \sqsubseteq C_{2}$ is implied by the axioms in $\mathcal{T}$, it is added to $\mathcal{T}$. Implication is defined in the usual way, i.e. $\mathcal{T} \models C_{1} \sqsubseteq C_{2}$ iff $\mathcal{S}\left(C_{1}\right) \subseteq \mathcal{S}\left(C_{2}\right)$ holds for every model of $\mathcal{T}$.

We further assume that the assertions in the ABox have been completed as well, i.e. whenever $C_{1}(o)$ is such an assertion and the axiom $C_{1} \sqsubseteq C_{2}$ is in $\mathcal{T}$, then also $C_{2}(o)$ is asserted in the ABox.

With these assumptions we can treat the ABox as a simple relational database with unary and binary relation symbols for the concepts and roles of the terminology $\mathcal{T}$, respectively. Then any query language for relational databases, calculus, algebra, SQL, fixed-point logic, DATALOG, etc. can be used to query a cloud. However, searching for services on the web may be done without exact knowledge of the terminology $\mathcal{T}$ of a cloud. In this case techniques from information retrieval with similarity vectors for keywords - in this case concept and role names - can be applied first to find a service matching a "similar" request up to some reliability factor. The details of this idea still have to be worked out.

\section{CONCLUSION}

In this paper we continued our work on foundations of (webbased) service-oriented systems by extending our ASM-based model of Abstract State Services (ASSs) to a model of clouds. We started from an understanding of a cloud as a federation of services, and addressed the problem of service discovery. We observed that for a service seeker to be able to discover a suitable service the service specification is not sufficient nor is a functional description by means of types, and pre- and post-conditions.

This leads to the requirement that a cloud should provide a description of its services by means of an ontology, which must enrich the functional service descriptions by keyword descriptions that describe the application area, and the available data and functions. This can be exploited to match the requests of a service seeker with the available services, and thus supports the semi-automatic selection of services. The reliability of such matches can be further increased by integrating clouds into trusted service brokers (TSBs) with publicised terminology used in the ontology.

Ontologies associated with clouds and TSBs, respectively, can be further extended by adding the specification of nonfunctional (aka "quality of service") properties such as response time, availability, reliability, price, etc. These properties can be used to optimise the selection of services for a particularly service-oriented system in case there are alternative solutions.

We intend to extend our research in various directions. As the model of ASSs is based on database transformations, we already know that all database transformations are captured (this was shown in [15]). It would thus be a natural consequence to investigate ASSs that are bound to a particular data model, e.g. relational databases or XML, and to tailor service specifications to available languages such as XQuery.

Furthermore, we need to explore the theory of the cloud model in more detail. Due to the incorporation of keyword descriptions a completeness result as for ASSs cannot be expected. However, solid results, how well an ontology for a particular application area can capture the service needs in that area would be desirable. The need for optimised service selection based on quality-of-service criteria constitutes another line of research to continue our present work. Finally, case studies applying the framework would be helpful to gain further insights.

\section{REFERENCES}

[1] M. Altenhofen, E. Börger, and J. Lemcke. An abstract model for process mediation. In K.-K. Lau and R. Banach, editors, Formal Methods and Software Engineering, 7th International Conference on Formal Engineering Methods (ICFEM 2005), volume 3785 of Lecture Notes in Computer Science, pages 81-95. Springer-Verlag, 2005.

[2] A. Alves et al. Web services business process execution language, version 2.0, 2007. OASIS Standard Committee, http://docs.oasisopen.org/wsbpel/2.0/wsbpel-v2.0.html.

[3] F. Baader et al., editors. The Description Logic Handbook: Theory, Implementation and Applications. Cambridge University Press, 2003.

[4] B. Benatallah, F. Casati, and F. Toumani. Representing, analysing and managing web service protocols. Data and Knowledge Engineering, 58(3):327-357, 2006.

[5] A. Blass and J. Gurevich. Abstract state machines capture parallel algorithms. ACM Transactions on Computational Logic, 4(4):578-651, 2003.

[6] E. Börger and R. Stärk. Abstract State Machines. Springer-Verlag, Berlin Heidelberg New York, 2003.

[7] M. R. Brenner and M. R. Unmehopa. Service-oriented architecture and web services penetration in next-generation networks. Bell Labs Technical Journal, 12(2):147-159, 2007.

[8] E. Christensen et al. Web services description language (WSDL) 1.1, 2001. http://www.w3c.org/TR/wsdl.

[9] J. Gurevich. Sequential abstract state machines capture sequential algorithms. ACM Transactions on Computational Logic, 1(1):77-111, 2000 .

[10] S. Kumaran et al. Using a model-driven transformational approach and service-oriented architecture for service delivery management. IBM Systems Journal, 46(3):513-530, 2007.

[11] H. Ma, K.-D. Schewe, B. Thalheim, and Q. Wang. Abstract state services. In I.-Y. Song et al., editors, Advances in Conceptual Modeling - Challenges and Opportunities, ER 2008 Workshops, volume 5232 of LNCS, pages 406-415. Springer-Verlag, 2008.

[12] H. Ma, K.-D. Schewe, B. Thalheim, and Q. Wang. A theory of dataintensive software services. (submitted for publication), 2009.

[13] M. P. Papazoglou and W.-J. van den Heuvel. Service oriented architectures: Approaches, technologies and research issues. VLDB Journal, 16(3):389-415, 2007.

[14] K.-D. Schewe and B. Thalheim. Conceptual modelling of web information systems. Data and Knowledge Engineering, 54(2):147-188, 2005.

[15] K.-D. Schewe and Q. Wang. A customised ASM thesis for database transformations, 2008. submitted for publication.

[16] Y. Tanaka. Meme Media and Meme Market Architectures. IEEE Press, Wiley-Interscience, USA, 2003.

[17] Web ontology language (OWL). http://www.w3c.org//OWL/. 